\title{
Sensory Phenomena in Tourette Syndrome: Their Role in Symptom Formation and Treatment
}

\author{
David C. Houghton • Matthew R. Capriotti • \\ Christine A. Conelea $\cdot$ Douglas W. Woods
}

Published online: 23 August 2014

(C) Springer International Publishing Switzerland 2014

\begin{abstract}
The primary symptoms of Tourette Syndrome (TS) are motor and vocal tics, but increasingly, researchers have examined the role of sensory phenomena in biobehavioral models of the disorder. These sensory phenomena involve tic-related premonitory urge sensations as well as potential abnormalities in the perceptual and behavioral experiences associated with external sensory input. As such, dysfunctional sensorimotor integration might represent a key facet of TS pathology. The current paper reviews the literature on sensory phenomena in tic disorders and highlights possible connections to TS symptoms and directions for future research.
\end{abstract}

Keywords Tourette syndrome $\cdot$ Chronic tic disorder · Tics · Neurodevelopmental disorders $\cdot$ Sensorimotor integration

\section{Introduction}

Tourette Syndrome (TS) is a neurodevelopmental condition characterized by repetitive motor and vocal tics [1] that begin

\footnotetext{
D. C. Houghton · D. W. Woods $(\bowtie)$

Department of Psychology, Texas A\&M University, 4235 TAMU, College Station, TX 77843, USA

e-mail: dowoods@tamu.edu

D. C. Houghton

e-mail: davidhoughton@tamu.edu

\section{R. Capriotti}

Department of Psychology, University of Wisconsin-Milwaukee, 224 Garland Hall, 2441 E. Hartford Ave, Milwaukee, WI 53211, USA

e-mail: capriot2@uwm.edu

\section{A. Conelea}

Bradley Hasbro Children's Research Center, Alpert Medical School of Brown University, One Hoppin Street, Providence, RI 02903, USA

e-mail: christine_conelea@brown.edu
}

during childhood and typically increase in severity through early adolescence [2]. Tics are repetitive, driven, and often rapid behaviors that appear to serve no obvious purpose; and can range from simple (e.g., hard blinking and grunting) to more complex and purposeful-looking actions (e.g., repeating words and touching or tapping objects).

Research investigating the biological underpinnings of TS has revealed structural and functional abnormalities within cortico-striatal-thalamo-cortical (CSTC) circuits [3]. Hyperexcitability within the motor cortex is thought to lead to increased urges to move and impaired movement (e.g., tics and poor movement inhibition), which is then believed to be reinforced by activity-dependent dopaminergic feedback [3]. Contemporary models of TS also account for the role of nonbiological variables $[4,5,6 \cdot 7 \cdot, 8]$. For instance, research indicates that behavioral (i.e., contextual) variables impact tic expression $[9,10]$, which suggests that tics may be maintained or shaped as a part of experience-dependent plasticity within implicated neural circuitry [11].

Furthering the notion that both neurobiological and behavioral variables are involved in TS pathology, research has discovered aberrant sensory phenomena in affected individuals. Both interoceptive sensory information and exteroceptive sensory feedback are instrumental in voluntary motor output $[12,13]$. Indeed, persons with TS demonstrate abnormalities in processing within these domains [14], which could result in dysfunctional sensorimotor integration and therefore affect motor control $\left[6^{\bullet}\right]$.

Given that sensory experiences affect tics, an understanding of how this occurs could provide a more complete understanding of the disorder and allow for treatments to be developed that target these processes. Most of the research on sensory issues related to tics has focused on premonitory urges (PMUs), but other sensory triggers for tics have been described, and research on the processing of sensory information in persons with TS has been conducted. This paper will 
provide examples of relevant sensory phenomena in TS and, where there is adequate evidence, suggest possible mechanisms by which sensory signals influence tics.

\section{Premonitory Urges (PMUs)}

Initial Research Findings. The most widely recognized of the sensory phenomena experienced by those with TS are PMUs, which are uncomfortable sensory experiences that precede tics and diminish following tic occurrence [15]. PMUs may occur in a specific region of the body (usually in the same area as the tic it precedes) or may be experienced as 'generalized' across the entire body [16]. Some patients also report experiencing obsessive-type thoughts (e.g., "Something is just not right") that accompany these physical sensations. However, PMUs are phenomenologically different from obsessions associated with obsessive-compulsive disorder (OCD), in that the latter are associated with anxious arousal and feared consequences, whereas PMUs tend to be associated with muscular tension and somatic discomfort [16].

Although the earliest published discussion of PMUs dates back over a century [17], modern research on PMUs has occurred mainly since the 1980s. In early studies, PMUs were defined rather narrowly (i.e., as focalized muscular tension) and were only found in a minority of TS patients $[18,19]$. However, when PMUs were defined more broadly to include a range of aversive somatic experiences preceding tics, studies showed that greater than $90 \%$ of TS patients aged 9 years and older reported some type of PMU $[8,15,18]$.

PMU prevalence among younger children is unclear, but limited data indicate that PMUs tend to onset about 3 years after tics first appear [15]. Reasons for this delay are currently unknown. Some have theorized that young children may experience sensations akin to PMUs, but lack the cognitive abilities needed to describe internal sensations and their relation to tics [8]. Indeed, preliminary evidence suggests that children (aged 8-11 years) have poor ability to identify interoceptive sensations [20].

Once thought to be a secondary phenomenological symptom associated with TS, PMUs are now believed to play a critical role in perpetuating tics [21]. After PMUs develop, they strengthen tics via a negative reinforcement process. Said another way, ticcing has the unique ability to attenuate PMUs, which increases the probability of tics occurring in the future. This notion was first suggested by Patrick [17] and more formally iterated by Evers and van de Wetering [4], who proposed a 'tension-reduction' model of TS. Indeed, a subsequent series of experimental studies have provided empirical support consistent with the tension-reduction, or negative reinforcement, hypothesis. For instance, two studies demonstrated that PMU strength increased during periods of reinforced tic suppression and decreased during breaks from suppression [22•, 23]. Additionally, Beetsma et al. [24] demonstrated that a simulated PMU produced discomfort and subsequent tic-like behavior in a sample of healthy adults. These findings suggest that severity of PMUs should correspond to tic severity, but research examining the correlation between these variables has produced mixed findings $[8$, 25-27], prompting the need for future investigations into the nature of this relationship. Nevertheless, considerable evidence supports the notion that PMUs perpetuate tics.

Factors Leading to Development of PMUs. Relatively little is known about the physiological substrates of PMUs. Three neuroimaging studies found heightened activation prior to tic onset in paralimbic and sensorimotor structures such as the supplementary motor area (SMA), anterior cingulate and insular cortex, and parietal operculum [7•,28, 29], particularly for spontaneous tics related to voluntary movements $[7 \cdot, 28]$. Data from these studies suggest that hyperactivation of the anterior cingulate and SMA may subserve the urge experience, similar to findings of studies examining urges to engage in other behaviors (e.g., scratching an itch, urinating when one's bladder is full) [30]. Other research has shown that electrical stimulation of the SMA produces tic-like movements, echophenomena, and urges to move in individuals without tics, adding evidence to support the role of SMA in PMUs and TS [31]. It is also possible that different phenomenological aspects of PMUs have different neurological underpinnings. For example, Wang et al. [7•] speculated that greater activity in the somatosensory cortex might underlie the sensory aspects of PMUs while greater activation of the amygdala/hippocampal complex may be associated with the emotional discomfort of urges.

Research has also examined the biopsychosocial factors contributing to the development of PMUs. Several crosssectional studies have suggested that certain contextual variables may contribute to PMU development. For example, aversive consequences (e.g., being teased or stared at for ticcing) $[32,33]$ or escape-related consequences to tics (e.g., stopping homework or not completing chores due to tic occurrence) $[10,34]$ have been found to be associated with urges. Supporting these findings are data showing that PMUs are positively correlated with indices of psychological symptomatology and impairment (e.g., anxiety and mood disorder symptoms, withdrawal, social impairment, and aggression) [8]. Furthermore, another study showed that PMUs are associated with activation of neural structures involved in negative affect and punishment-based learning [7]. Research has yet to explain why contextual variables influence tics, but, from a behavioral perspective, it is possible that repeated pairing of the urge with certain aversive and desirable experiences might modulate urge severity through conditioning processes. 
PMUs in Treatment. Behavioral models posit that inhibiting tics in the presence of urges will result in habituation to urges, thus resulting in fewer tics. Indeed, several studies have demonstrated that exposure to PMUs while inhibiting tics results in urge reductions [35, 36], and behavioral interventions utilizing these techniques therapeutically have been found to be effective. For example, both Exposure and Response Prevention and Habit Reversal Training (HRT) have been shown to significantly reduce tic severity $[5,35,37,38]$. Two recently conducted randomized clinical trials of Comprehensive Behavioral Intervention for Tics, which includes HRT and other behavioral techniques, have shown the treatment to be effective for both adults and children [39, 40].

Although it is not yet clear exactly how changes in PMUs relate to treatment outcome, a number of studies suggest that both psychological and pharmacological interventions may alter PMUs and/or patients' responses to them. Behavior therapy techniques are thought to facilitate habituation to PMUs, but only one experimental trial has verified the predicted reductions [36], whereas no other studies have reported reductions in PMU severity produced by behavior therapy. Interestingly, one treatment not explicitly meant to target PMUs, botulinum neurotoxin, has shown consistent urge and tic reductions [41-43], suggesting that pharmacologically facilitated tic suppression can also affect urges. Nevertheless, a study examining ecopipam, a dopamine antagonist, found reductions in tics but no effects on PMUs [44], suggesting that treatments need not necessarily target the urge directly. Combined, these studies suggest that tic reduction can be accomplished via multiple processes that do not always impact PMUs, and future research should measure PMU effects associated with a multitude of treatment modalities. Also, it remains to be seen if treatments that target PMUs (i.e., behavior therapy) actually involve habituation or if other processes modulate the urge-tic relationship.

\section{Other Sensory Issues in TS}

Along with the research on PMUs, scientific inquiries focused on other aspects of sensory functioning in persons with TS have also been conducted. Whether and how these other sensory factors trigger tics is somewhat unclear, but growing evidence suggests that various abnormal sensory experiences are associated with TS. In the following sections, we review several emerging areas of TS research, such as reports of tics that are elicited by external stimuli as well as additional abnormal sensory phenomena.

Ticcing in Response to Exteroceptive Stimuli. It has long been noted that some tics may be performed in response to external stimuli. For instance, Gilles de la Tourette [45] first documented that tics can sometimes take the form of mirroring others' movements and/or vocalizations, otherwise known as echopraxia and echolalia, respectively. Researchers have also documented instances of tics that are performed repeatedly in response to more specific environmental cues, including cases of TS where tics are exacerbated during exposure to specific visual, auditory, and tactile sensory stimuli (e.g., witnessing others cough, hearing certain words and sounds, exposure to hot temperatures) [46-49].

Abnormal Sensory Experiences. Research has uncovered several ways in which persons with TS react abnormally to various sensory inputs, suggesting that a range of dysfunctional sensory processes may have a role in TS pathology. In our review of the extant literature, three thematic categories emerged: compulsive self-evaluation of behavior and need for actions to feel 'just right'; heightened interoceptive awareness; and hypersensitivity and intolerance to exteroceptive stimuli. The following sections detail relevant research findings in these areas.

Individuals with TS have demonstrated a tendency to compulsively evaluate their own behavior, thus needing to repeat actions until 'successful' completion. In contrast to normal behavioral evaluation, wherein individuals use afferent sensory inputs to determine whether actions were completed as desired [50] or if actions need to be inhibited due to negative feedback [12], disturbances in sensory feedback might create a situation where "sensory overflow" [51] results in obsessive concern for behaviors feeling, looking, or sounding 'just right,' 'even,' or 'complete.' As such, several studies have investigated rates of 'just right' phenomena in TS [52-55], reporting rates between $30 \%$ and $90 \%$. Furthermore, Biermann-Ruben et al. [51] found that adults with TS showed increased neural activity in areas associated with sensory feedback (i.e., contralateral primary somatosensory cortex) following self-initiated and reactive movements, potentially shedding light on the neurological basis for 'just right' experiences.

Individuals with TS also appear to demonstrate heightened interoceptive awareness. An early study on this topic found that self-reported number of tics was positively correlated with attentiveness to bodily sensations [56]. Likewise, Eddy et al. [57] found that adults with TS reported increased awareness of internal states compared with non-TS matched controls. Finally, Sutherland Owens et al. [58] assessed five types of disordered sensory phenomena (physical sensations, "just right" perceptions, feelings of incompleteness, energy, and "urges only") using the University of São Paulo Sensory Phenomena Scale (USP-SPS), and found that USP-SPS total scores were positively correlated with PMUs.

Several studies have shown that individuals with TS selfreport abnormal reactions to exteroceptive stimuli, such as 
heightened sensory sensitivity/intolerance $[59,60]$. To measure this phenomenon more objectively, Sutherland Owens et al. [58] used a self-report measure (the Sensory Gating Inventory [61]) containing items such as, "At times I have feelings of being flooded by sounds," and, "I have more trouble concentrating than others have." Results showed that adults with TS demonstrated problems with perceiving increases in stimulus intensity, concentrating and focusing on stimuli, and excluding irrelevant background stimuli as compared to healthy controls. In another recent study [62], sensory intolerance - defined as experiencing high levels of distress in response to ordinary external stimuli (e.g., tags on clothes, elastic sock bands, sounds of others chewing food) - was studied in a community-based internet sample. Using latent class analysis, the researchers differentiated those who were sensory intolerant and those who were not. Those in the sensory intolerant group were statistically more likely to report a lifetime diagnosis of OCD (59\% vs $26 \%)$ and lifetime history of tics ( $17 \%$ vs $6 \%$ ), suggesting that sensory intolerance might be associated with ritualistic and repetitive behaviors.

\section{Possible Explanations for Sensory Phenomena beyond PMUs}

Research reviewed above has offered insights into sensory features associated with TS, but the specific role of these phenomena in the disorder's etiology remains unclear. The relationship of sensory phenomena to tic disorders is a complex dynamic, and might be better understood through the integration of multiple perspectives on TS pathology. Although the current literature does not connect the sensory phenomena observed in TS directly to underlying neurobiological etiologies, we suggest several possibilities in the following sections.

Enhanced Perceptual Abilities. One possible explanation for the effects of external environmental stimuli on persons with tics could be that individuals with TS possess enhanced sensory sensitivity. However, mixed data on abnormalities in somatosensory evoked potentials, the neural signatures of sensory input, have been reported in TS [51, 63, 64]. In addition, Belluscio et al. [59] found no increased ability to detect tactile stimuli, even though participants with TS selfreported more perceived sensory sensitivity than controls. Therefore, it is possible that abnormal reactions to stimuli are not due to enhanced perceptual abilities but may reflect altered central processing or differential affective or behavioral response to equivalent sensory input. It is also possible that sensory sensitivity is common in psychiatric populations at large, but not necessarily more so in individuals with TS.
Indeed, data suggest sensory sensitivity is associated with a variety of internalizing and externalizing disorders [65].

Neurobiological Dysfunction. Sensorimotor integration, a process by which the central nervous system integrates sensory input to plan for motor outputs [14], is essential for efficient movement. Research suggests that the basal ganglia are involved in the selective inhibition of various sensory inputs, and, along with dysfunctional sensorimotor cortex projections, abnormal sensorimotor integration could lead to disruptions in movement control, possibly representing a key facet of TS pathology [14, 66, 67].

One mechanism that might explain part of the proposed deficits in sensorimotor integration is sensory gating. Sensory gating is a neurological process whereby redundant and unnecessary stimuli are filtered and prevented from processing within the central nervous system. An inability to effectively filter extraneous forms of sensory inputs might lead to a variety of abnormalities, such as sensory intolerance, sensitivity, and heightened interoceptive awareness. Indeed, deficits in sensory gating abilities have been identified in persons with TS. For instance, measurement of sensory gating generally focuses on prepulse inhibition (PPI); a measure of reduced inhibition of a startle response (e.g., blinking) when a stimulus, or prepulse, occurs shortly before (30 to $500 \mathrm{~ms}$ ) a startle stimulus (e.g., a loud tone). Research has demonstrated reduced PPI in adults and children with TS [68-71]. Furthermore, animal models [72] and studies examining synaptic inhibition [73] have supported the notion that sensory inputs in TS have increased access to motor output. Nevertheless, the specific relationship between sensory gating processes and sensory phenomena in tics has yet to be examined and warrants further investigation.

\section{Conclusions}

Existing research reviewed herein suggests that (a) PMUs are a core feature of TS that affect tics strongly, (b) PMU reduction may be one mechanism by which certain behavioral and pharmacological treatments reduce tics, and (c) a host of other aberrant sensory phenomena are observed in TS that might be explained by dysfunctional sensory detection or gating processes.

Given the lack of a clear connection between sensory phenomena and tics (other than with PMUs), our conclusions regarding the role of sensory issues in TS pathology, and how these variables could be targets of future treatments, remain limited. It is believed that the underlying neuropathology of CSTC circuits in TS results in abnormal sensorimotor integration, which potentially explains why tics are often triggered by sensory experiences and why persons with TS show 
abnormal reactions to benign stimuli. However, development of a comprehensive model for this system requires far more empirical attention. Several aims for future work in this area are described below.

First, research has occurred at different levels of analysis and rarely links to tics themselves. Future investigations should pursue integrative methodology connecting neurobiological functioning, sensory/perceptual experiences, and tics. Second, the literature is replete with vague and inconsistent construct/operational definitions of sensory phenomena in TS (e.g., hypervigilance vs hypersensitivity). Future research should establish clearly defined operational definitions of phenomena with better construct and discriminant validity. Third, good assessment tools exist for PMUs [8] and some biological facets of sensory processing (e.g., PPI, threshold detection), but fewer empirically sound measures exist for 'just right' experiences and heightened sensitivity. Finally, it is unclear whether these phenomena are specific to TS, as many are implicated in other forms of psychopathology (e.g., OCD, anxiety, autism spectrum, and attention deficit/ hyperactivity disorder), which are frequently seen concurrently with TS. While it might be expected that deficits associated with CSTC dysfunction would express themselves transdiagnostically, future research should examine these features carefully using interdisciplinary approaches in order to parse out whether or not they are directly related to TSspecific symptomatology.

\section{Compliance with Ethics Guidelines}

Conflict of Interest David C. Houghton, Matthew R. Capriotti, Christine A. Conelea, and Douglas W. Woods declare that they have no conflict of interest.

Human and Animal Rights and Informed Consent This article does not contain any studies with human or animal subjects performed by any of the authors.

\section{References}

Papers of particular interest, published recently, have been highlighted as:

- Of importance

1. American Psychiatric Association. Diagnostic and Statistical Manual of Mental Disorders. 5th ed. American Psychiatric Association; 2013.

2. Leckman JF, Zhang H, Vitale A, et al. Course of tic severity in Tourette syndrome: the first two decades. Pediatrics. 1998;102(1 Pt 1):14-9.

3. Albin RL, Mink JW. Recent advances in Tourette syndrome research. Trends Neurosci. 2006;29(3):175-82. doi:10.1016/j.tins. 2006.01.001.
4. Evers RA, van de Wetering BJ. A treatment model for motor tics based on a specific tension-reduction technique. J Behav Ther Exp Psychiatry. 1994;25(3):255-60.

5. Himle MB, Woods DW, Piacentini JC, Walkup JT. Brief review of habit reversal training for Tourette syndrome. J Child Neurol. 2006;21(8):719-25.

6. Patel N, Jankovic J, Hallett M. Sensory aspects of movement disorders. Lancet Neurol. 2014;13(1):100-12. A review of shared deficits in sensorimotor integration between Tourette Syndrome and other movement disorders, highlighting implicated neural systems and connections to symptomatology.

7. Wang Z, Maia TV, Marsh R, Colibazzi T, Gerber A, Peterson BS. The neural circuits that generate tics in Tourette's syndrome. Am J Psychiatry. 2011;168(12):1326-37. fMRI study of individuals with Tourette Syndrome and healthy controls demonstrating hyperactivity in cortico-striato-thalamo-cortical structures, providing evidence that these circuits might be implicated in the underlying neuropathology of this disorder.

8. Woods DW, Piacentini J, Himle MB, Chang S. Premonitory Urge for Tics Scale (PUTS): initial psychometric results and examination of the premonitory urge phenomenon in youths with tic disorders. J Dev Behav Pediatr. 2005;26(6):397-403.

9. Conelea CA, Woods DW. The influence of contextual factors on tic expression in Tourette's syndrome: a review. J Psychosom Res. 2008;65(5):487-96.

10. Himle MB, Capriotti MR, Hayes LP, et al. Variables associated with tic exacerbation in children with chronic tic disorders. Behav Modif. 2014. doi:10.1177/0145445514531016.

11. Graybiel AM. Habits, rituals, and the evaluative brain. Ann Rev Neurosci. 2008;31(1):359-87.

12. Nijs J, Daenen L, Cras P, Struyf F, Roussel N, Oostendorp RAB. Nociception affects motor output: a review on sensory-motor interaction with focus on clinical implications. Clin J Pain. 2012;28(2):175-81.

13. Seki K, Fetz EE. Gating of sensory input at spinal and cortical levels during preparation and execution of voluntary movement. J Neurosci. 2012;32(3):890-902.

14. Abbruzzese G, Berardelli A. Sensorimotor integration in movement disorders. Mov Disord. 2003;18(3):1-10.

15. Leckman JF, Walker DE, Cohen DJ. Premonitory urges in Tourette's syndrome. Am J Psychiatry. 1993;150(1):98-102.

16. Miguel EC, Coffey BJ, Baer L, Savage CR, Rauch SL, Jenike MA. Phenomenology of intentional repetitive behaviors in obsessivecompulsive disorder and Tourette's disorder. J Clin Psychiatry. 1995;56(6):246-55.

17. Patrick HT. Convulsive Tic. JAMA. 1905;44(6):437-42.

18. Montgomery MA, Clayton PJ, Friedhoff AJ. Psychiatric illness in Tourette syndrome patients and first-degree relatives. Adv Neurol. 1982;35:335-9.

19. Shapiro AK, Shapiro ES, Young JG, Feinberg TE. Gilles de la Tourette Syndrome. New York: Raven Press; 1988.

20. Eley TC, Stirling L, Ehlers A, et al. Heart-beat perception, panic/ somatic symptoms and anxiety sensitivity in children. Behav Res Ther. 42(4):439-448.

21. Fahn S, Jankovic J, Hallett M. Principles and Practice of Movement Disorders. Elsevier Health Sciences; 2011.

22. Capriotti MR, Brandt BC, Turkel JE, Lee H-J, Woods DW. Negative reinforcement and premonitory urges in youth with Tourette syndrome: an experimental evaluation. Behav Modif. 2014. doi:10.1177/0145445514531015. An experimental study examining the relationship between tics and premonitory urges during tic suppression, providing clear evidence for the 'tension reduction,' or negative reinforcement hypothesis.

23. Himle MB, Woods DW, Conelea CA, Bauer CC, Rice KA. Investigating the effects of tic suppression on premonitory urge ratings in children and adolescents with Tourette's syndrome. Behav Res Ther. 2007;45(12):2964-76. 
24. Beetsma DJV, van den Hout MA, Engelhard IM. Does repeated ticking maintain tic behavior? An experimental study of eye blinking in healthy individuals. Behav Neurol. 2013;27:1-9.

25. Crossley E, Seri S, Stern JS, Robertson MM, Cavanna AE. Premonitory urges for tics in adult patients with Tourette syndrome. Brain Dev. 2014;36(1):45-50.

26. Reese HE, Scahill L, Peterson AL, et al. The premonitory urge to tic: measurement, characteristics, and correlates in older adolescents and adults. Behav Ther. 2014;45(2):177-86.

27. Steinberg T, Shmuel Baruch S, Harush A, et al. Tic disorders and the premonitory urge. J Neural Transm. 2009;117(2):277-84.

28. Bohlhalter S, Goldfine A, Matteson S, et al. Neural correlates of tic generation in Tourette syndrome: an event-related functional MRI study. Brain. 2006;129(Pt 8):2029-37.

29. Peterson BS, Skudlarski P, Anderson AW, et al. A functional magnetic resonance imaging study of tic suppression in Tourette syndrome. Arch Gen Psychiatry. 1998;55(4):326-33.

30. Jackson SR, Parkinson A, Kim SY, Schüermann M, Eickhoff SB. On the functional anatomy of the urge-for-action. Cogn Neurosci. 2011;2(3-4):227-43.

31. Finis J, Enticott PG, Pollok B, Münchau A, Schnitzler A, Fitzgerald PB. Repetitive transcranial magnetic stimulation of the supplementary motor area induces echophenomena. Cortex. 2013;49(7): 1978-82.

32. Capriotti MR, Espil FM, Conelea CA, Woods DW. Environmental factors as potential determinants of premonitory urge severity in youth with Tourette syndrome. J Obsess-Compul Relat Disord. 2013;2(1):37-42.

33. Zinner SH, Conelea CA, Glew GM, Woods DW, Budman CL. Peer victimization in youth with Tourette syndrome and other chronic tic disorders. Child Psychiatry Hum Dev. 2011;43(1):124-36.

34. Capriotti MR, Piacentini J, Himle MB, et al. Assessing environmental consequences of ticcing in youth with chronic tic disorders: The Tic Accommodation and Reactions Scale. Children's Health Care. In Press.

35. Hoogduin K, Verdellen C, Cath D. Exposure and response prevention in the treatment of Gilles de la Tourette's syndrome: four case studies. Clin Psychol Psychother. 1997;4(2):125-35.

36. Verdellen CWJ, Hoogduin CAL, Kato BS, Keijsers GPJ, Cath DC, Hoijtink HB. Habituation of premonitory sensations during exposure and response prevention treatment in Tourette's syndrome. Behav Modif. 2008;32(2):215-27.

37. Cook CR, Blacher J. Evidence-based psychosocial treatments for tic disorders. Clin Psychol Sci Pract. 2007;14:252-67.

38. Verdellen CWJ, Keijsers GP, Cath DC, Hoogduin CA. Exposure and response prevention versus habit reversal in Tourette's syndrome: a controlled study. Behav Res Ther. 2004;42(5):501-11.

39. Piacentini J, Woods DW, Scahill L, et al. Behavior therapy for children with Tourette disorder. JAMA. 2010;303(19):1929-37.

40. Wilhelm S, Peterson AL, Piacentini J, et al. Randomized trial of behavior therapy for adults with Tourette syndrome. Arch Gen Psychiatry. 2012;69(8):795-803.

41. Jankovic J. Botulinum toxin in the treatment of dystonic tics. Mov Disord. 1994;9(3):347-9.

42. Kwak CH, Hanna PA, Jankovic J. Botulinum toxin in the treatment of tics. Arch Neurol. 2000;57(8):1190-3.

43. Marras C, Andrews D, Sime E, Lang AE. Botulinum toxin for simple motor tics. Neurology. 2001;56(5):605-10.

44. Gilbert DL, Budman CL, Singer HS, Kurlan R, Chipkin RE. A D1 receptor antagonist, ecopipam, for treatment of tics in Tourette syndrome. Clin Neuropharmacol. 2014;37(1):26-30.

45. Tourette GGDL. Étude sur une affection nerveuse caracterisée par de l'incoordination motrice accompagnée d'écholalie et de coprolalie (jumping, latah, myriachit). Arch Neurol. 1885;9(1942):158-200.
46. Commander M, Corbett J, Prendergast M, Ridley C. Reflex tics in two patients with Gilles de la Tourette syndrome. Br J Psychiatry. 1991;159(6):877-9.

47. Eapen V, Moriarty J, Robertson MM. Stimulus induced behaviours in Tourette's syndrome. J Neurol Neurosurg Psychiatry. 1994;57(7): $853-5$.

48. Lombroso PJ, Mack G, Scahilll L, King RA, Leckman JF. Exacerbation of Gilles de la Tourette's syndrome associated with thermal stress: A family study. Neurology. 1984;41(12):1984.

49. Webber TA, Johnson PL, Storch EA. Pediatric misophonia with comorbid obsessive-compulsive spectrum disorders. Gen Hosp Psychiatry. 2014;36(2):231-2.

50. Proske U, Gandevia SC. The kinaesthetic senses. J Physiol. 2009;587(17):4139-46.

51. Biermann-Ruben K, Miller A, Franzkowiak S, et al. Increased sensory feedback in Tourette syndrome. NeuroImage. 2012;63(1): $119-25$.

52. Leckman JF, Walker DE, Goodman WK, Pauls DL, Cohen DJ. "Just right" perceptions associated with compulsive behavior in Tourette's syndrome. Am J Psychiatry. 1994;151(5):675-80.

53. Miguel EC. do Rosario Campos MC, Prado HS, et al. Sensory phenomena in obsessive-compulsive disorder and Tourette's disorder. J Clin Psychiatry. 2000;61(2):150-6.

54. Neal M, Cavanna AE. "Not just right experiences" in patients with Tourette syndrome: complex motor tics or compulsions? Psychiatry Res. 2013;210(2):559-63.

55. Worbe Y, Mallet L, Golmard J-L, et al. Repetitive behaviours in patients with Gilles de la Tourette syndrome: tics, compulsions, or both? PLoS ONE. 2010;5(9):e12959.

56. Woods DW, Miltenberger RG, Flach AD. Habits, tics, and stuttering: prevalence and relation to anxiety and somatic awareness. Behav Modif. 1996;20(2):216-25.

57. Eddy CM, Rickards HE, Cavanna AE. Physiological awareness is negatively related to inhibitory functioning in Tourette syndrome. Behav Modif. 2013. doi:10.1177/0145445513504431.

58. Sutherland Owens AN, Miguel EC, Swerdlow NR. Sensory gating scales and premonitory urges in Tourette syndrome. Sci World J. 2011;11:736-41.

59. Belluscio BA, Jin L, Watters V, Lee TH, Hallett M. Sensory sensitivity to external stimuli in Tourette syndrome patients. Mov Disord. 2011;26(14):2538-43.

60. Cohen DJ, Leckman JF. Sensory phenomena associated with Gilles de la Tourette syndrome. J Clin Psychiatry. 1992;53(9):319-23.

61. Hetrick WP, Erickson MA, Smith DA. Phenomenological dimensions of sensory gating. Schizophr Bull. 2012;38(1):178-91.

62. Taylor S, Conelea CA, McKay D, Crowe KB, Abramowitz JS. Sensory intolerance: latent structure and psychopathologic correlates. Compr Psychiatry. 2014. doi:10.1016/j.comppsych.2014.03. 007.

63. Krumholz A, Singer HS, Niedermeyer E. Electrophysiological studies in Tourette's syndrome. Ann Neurol. 1983;14(6):638-41.

64. Syrigou-Papavasiliou A, Verma NP, LeWitt PA. Sensory evoked responses in Tourette syndrome. Clin Electroencephalogr. 1988;19(2):108-10.

65. Carter AS, Ben-Sasson A, Briggs-Gowan MJ. Sensory overresponsivity, psychopathology, and family impairment in schoolaged children. J Am Acad Child Adolesc Psychiatry. 2011;50(12): 1210-9.

66. Kaji R, Murase N. Sensory function of basal ganglia. Mov Disord. 2001;16(4):593-4.

67. Rajagopal S, Seri S, Cavanna AE. Premonitory urges and sensorimotor processing in Tourette syndrome. Behav Neurol. 2013;27:110.

68. Castellanos FX, Fine EJ, Kaysen D, Marsh WL, Rapoport JL, Hallett M. Sensorimotor gating in boys with Tourette's syndrome 
and ADHD: preliminary results. Biol Psychiatry. 1996;39(1):3341.

69. Smith SJ, Lees AJ. Abnormalities of the blink reflex in Gilles de la Tourette syndrome. J Neurol Neurosurg Psychiatry. 1989;52(7): 895-8.

70. Swerdlow NR, Karban B, Ploum Y, et al. Tactile prepuff inhibition of startle in children with Tourette's syndrome: in search of an "fMRI-friendly" startle paradigm. Biol Psychiatry. 2001;50(8): 578-85.
71. Zebardast N, Crowley MJ, Bloch MH, et al. Brain mechanisms for prepulse inhibition in adults with Tourette syndrome: initial findings. Psychiatry Res. 2013;214(1):33-41.

72. Swerdlow NR, Sutherland AN. Using animal models to develop therapeutics for Tourette Syndrome. Pharmacol Ther. 2005;108(3): 281-93.

73. Orth M, Münchau A. Transcranial magnetic stimulation studies of sensorimotor networks in Tourette syndrome. Behav Neurol. 2013;27:1-9. 